\title{
Thermal Analysis and Experimental Study of End-Pumped Nd: YLF Laser at $1053 \mathrm{~nm}$
}

\author{
R. M. EL-AGMY ${ }^{1 *}$ and N. AL-HOSINY ${ }^{2,3}$ \\ ${ }^{1}$ Department of Physics, Faculty of Science, Helwan University 11792-Helwan, Egypt \\ ${ }^{2}$ Department of Physics, Faculty of Science, Aljouf University, Sakkaka, Saudi Arabia \\ ${ }^{3}$ Department of Physics, Faculty of Science, Taif University, Taif, Saudi Arabia \\ ${ }^{*}$ Corresponding author: R. M. EL-AGMY_ E-mail: Redaagmy@science.helwan.edu.eg
}

\begin{abstract}
We have numerically analyzed the thermal effects in Nd: YLF laser rod. The calculations of temperature and stress distributions in the Nd: YLF laser rod was performed with finite element (FE) simulations. The calculations showed that the laser rod could be pumped up to a power of $40 \mathrm{~W}$ without fracture caused by thermal stress. The calculated thermal lens power of thermally induced lens in Nd: YLF ( $\sigma$-polarization) laser rod was analyzed and validated experimentally with two independent techniques. A Shack-Hartmann wavefront sensor and a Mach-Zehnder interferometer were used for direct measurements of focal thermal lens at different pump powers. The obtained measurements were coinciding with the FE simulations.
\end{abstract}

Keywords: Solid state lasers; thermally induced lens; wavefront sensors

Citation: R. M. EL-AGMY and N. AL-HOSINY, "Thermal Analysis and Experimental Study of End-Pumped Nd: YLF Laser at 1053 nm," Photonic Sensors, 2017, 7(4): 329-335.

\section{Introduction}

All diode pumped solid state (DPSS) lasers are of great interest for a number of industrial applications and high-power with good quality laser beams is needed for many purposes. Neodymium (Nd) doped different hosts such as yttrium aluminum garnet (YAG), glass, and yttrium lithium fluoride (YLF) emitting around $(1.047 \mu \mathrm{m}-1.053 \mu \mathrm{m})$ have been used for such applications. In DPSS lasers with end-pumped geometry, excellent efficiencies can be obtained along with a good modal selection, due to the optimal spatial matching of the pumping beam with the fundamental mode of the laser resonator [1-2]. In such geometries, power extraction is ultimately limited by consistent thermal distortions arising in the active medium even for relatively low pumping powers [3]. Thermal distortions of the active medium produce deleterious effects such as thermal lensing, depolarization, and bifocusing effects. Nd: YLF exhibits a strong natural birefringence, which overwhelms the thermally induced birefringence and thus eliminates thermal depolarization problems commonly encountered with isotropic media such as Nd: YAG [4]. Also, Nd: YLF has a negative $d n / d T$ which can over-power the positive thermal lensing from crystal end bulging resulting in a weak negative thermal lens [5]. However, Nd: YLF suffers a low thermally-induced stress fracture limit [6], restricting its power-scalability. Thermal effects in end and side pumped solid state lasers have been widely investigated since 1970 by Kochner to date [7-10], however, for our materials of interest Nd: YLF only

Received: 3 March 2017 / Revised: 5 July 2017

(C) The Author(s) 2017. This article is published with open access at Springerlink.com

DOI: $10.1007 / \mathrm{s} 13320-017-0412-6$

Article type: Regular 
few works have been reported [11-14]. This paper focuses on the analysis of thermal effect in Nd: YLF laser rods and its lasing characteristics at $1053 \mathrm{~nm}$ $\sigma$-polarization (electric field $\mathrm{E} / / c$-axis) rather than $1074 \mathrm{~nm} \pi$-polarization (electric field $\mathrm{E} \perp c$-axis). In this work, the temperature distributions as well as stress distributions were calculated with finite element (FE) simulations by using LASCAD software [15] in end pumped Nd: YLF laser rod. The thermal focal length was calculated, and the resonator design was carried out considering the value-change of thermal focal length of Nd: YLF crystal versus the pump powers. In experiment, thermal focal lengths of $\mathrm{Nd}$ : YLF laser rod at different pump powers were measured by using the Mach-Zehnder interferometer and Shack-Hartmann wavefront sensor. The obtained experimental measurements were agreed with the FE simulations.

\section{Finite element simulation results and discussion}

\subsection{Temperature and stress distributions in end-pumped Nd: YLF laser rod}

According to laboratory availability, we have simulated the Nd: YLF laser rod with lengths of $10 \mathrm{~mm}$ and $4 \mathrm{~mm}$ in diameter doped with $0.7 \%$ (at.) $\mathrm{Nd}^{+3}$. This low dopant concentration helped reduce the fracture risk of Nd: YLF laser rod and possible upconversion processes instead of the typical $1 \%-1.2 \%$ [13]. The rod was pumped from one end at the wavelength of $805 \mathrm{~nm}$. The Nd: YLF rod was water-cooled $\left(T_{\text {coolant }}=291 \mathrm{~K}\right)$, the front and back faces were in contact with air, and the heat transfer coefficients for water and air cooling were set to be $h=2.0 \quad \mathrm{~W} \cdot \mathrm{cm}^{-2} \cdot \mathrm{K}^{-1} \quad$ and $\quad h=0.005 \quad \mathrm{~W} \cdot \mathrm{cm}^{-2} \cdot \mathrm{K}^{-1}$, respectively [15]. The temperature dependence of the heat conductivity was taken into account. An additional concern in reducing pump intensity while maintaining diffraction limited fundamental mode oscillation was that the Nd: YLF radius was much larger ( 3 times [16-17]) than the pump radius. The Nd: YLF parameters used in the model were given in Table 1. We assumed a uniform pump configuration with pump radius of $300 \mu \mathrm{m}$ to avoid asymmetry.

Table $1 \mathrm{Nd}$ : YLF parameters used in the finite-element analysis [18].

\begin{tabular}{ccc}
\hline Refractive index & $\sigma$ & 1.448 \\
\hline Thermo-optical coefficient $d n / d T\left(10^{-6} / \mathrm{K}\right)$ & $\sigma$ & \multicolumn{2}{c}{-2} \\
Heat conductivity $(\mathrm{W} / \mathrm{mK})$ & \multicolumn{2}{c}{6.3} \\
Thermal expansion coefficient $\left(10^{-6} / \mathrm{K}\right)$ z-axis & \multicolumn{2}{c}{8.3} \\
Young's modulus $(\mathrm{GPa})$ & \multicolumn{2}{c}{85} \\
Poisson's ratio & \multicolumn{2}{c}{0.33} \\
Percent of absorbed pump power & $85 \%$ \\
Fraction of absorbed power which is converted to & $32 \%$ \\
heat under lasing condition [14] & $32 \%$ \\
\hline
\end{tabular}

2.2 Temperature and stress distributions in end-pumped Nd: YLF laser rod

Figure 1 shows the calculated temperature distribution for a cross-section through the Nd: YLF laser rod, which was pumped at $30 \mathrm{~W}$ from on end. The pump beam was focused at a $3 \mathrm{~mm}$ from the rod end to avoid bulging from the end face of the rod. The end bugling was complex for crystals which had different expansion coefficients for $a$ (or $y$ ) and $c$ (or $x$ ) axis in Nd: YLF rod. The detailed analysis of temperature distributions were indicated by different colors. The highest temperature was found where the pump beam entered the Nd: YLF rod, which also indicated where most of the pump photons were absorbed. It also decreased in the propagation direction as the pump was absorbed. The nonuniform temperature distribution resulted in mechanical stress and strain.

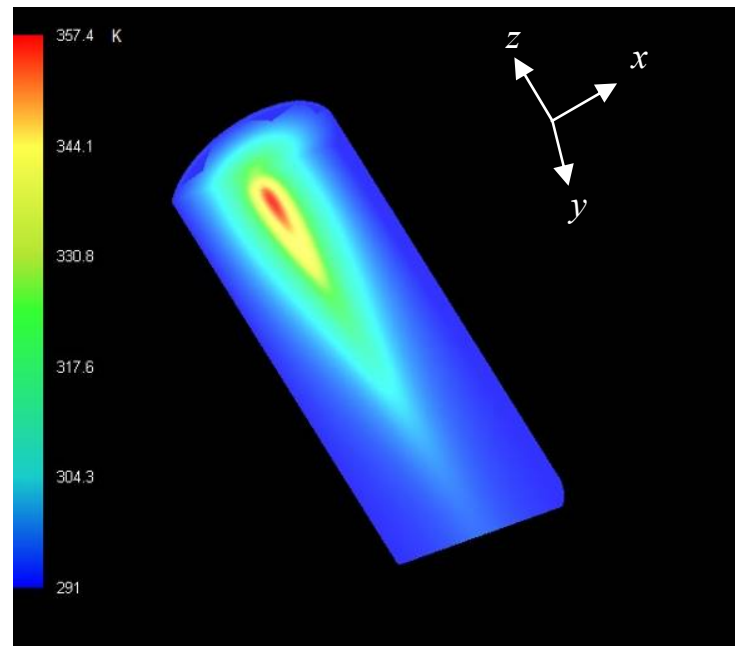

Fig. 1 Simulated temperature distribution in end pumped $\mathrm{Nd}$ : YLF rod of $4 \mathrm{~mm}$ diameter and $10 \mathrm{~mm}$ in length, pumped at $30 \mathrm{~W}$. 
Figure 2 shows the FE simulation results of stress intensity for Nd: YLF laser rod for the same conditions. The real amount and the spatial distribution of the stress intensity depended on the temperature distribution as well as on the material constants. The highest stress intensity was clear on the central top and bottom positions of the crystal. We could therefore expect thermally induced crack formation to start from these positions where the stress was shown to be high.

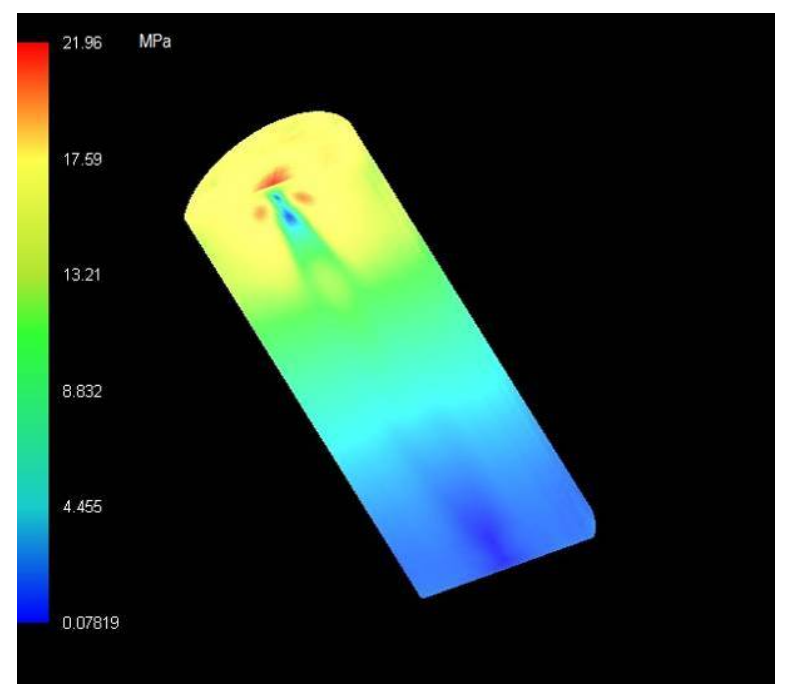

Fig. 2 Simulated stress intensity $(\mathrm{MPa})$ in end pumped $\mathrm{Nd}$ : YLF rod of $4 \mathrm{~mm}$ diameter and $10 \mathrm{~mm}$ in length, pumped at $30 \mathrm{~W}$.

Figure 3 shows plots of the maximum temperature increase in the center of the Nd: YLF rod and the maximum stress intensity with different

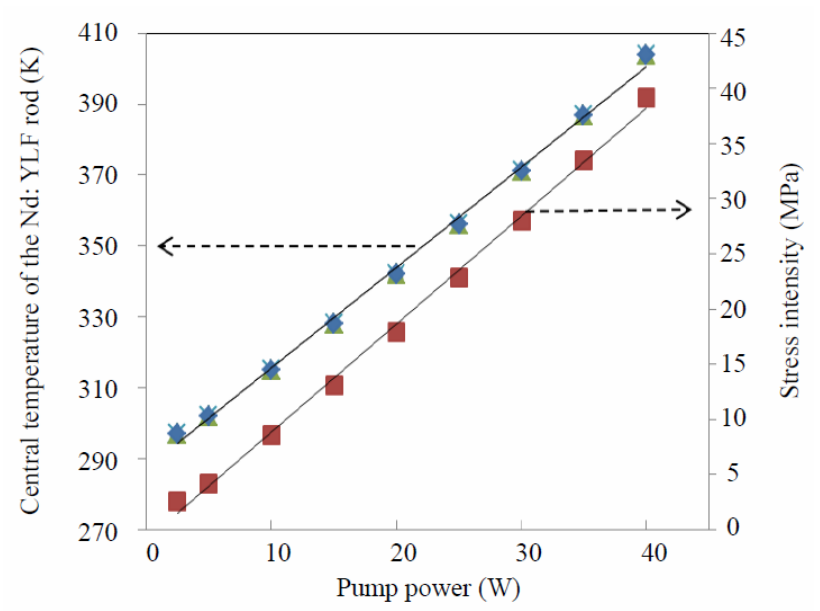

Fig. 3 Calculated maximum temperature and maximum stress intensity in the Nd: YLF laser rod for pump power ranged $2.5 \mathrm{~W}-40 \mathrm{~W}$. pump powers. As can be seen in Fig. 3, a linear increase in the central temperature as well as maximum stress intensity as a function of pump power was evident. The fracture limit of Nd: YLF laser rod was in the range of $32 \mathrm{MPa}-40 \mathrm{MPa}$ [19]. The Nd: YLF laser rod with above geometries could pump up to $(\sim 40 \mathrm{~W})$ before fracture occurred.

\subsection{Thermally induced lens and resonator stability}

The resulted temperature gradient from the axis to the surface was responsible also for the creation of thermally induced lens in the Nd: YLF laser rod. As mentioned above YLF crystal was a naturally birefringent crystal and had negative contribution to the thermally induced lens due to negative $d n_{\sigma, \pi d d T}$ for both polarization directions. The thermal lens in the $\sigma$-polarisation for laser transition at $1053 \mathrm{~nm}$ was about 2.3 weaker than $\pi$-polarisation for laser transition at $1047 \mathrm{~nm}$ [14]. It was concluded that $1053 \mathrm{~nm} \mathrm{Nd}$ : YLF exhibited a netto positive thermal lens at high pump powers, which were attributed to the positive lensing from end-face bulging being slightly more pronounced than the negative one [13, 15]. However, the presence of stronger negative lens in the ( $\pi$-polarization) direction in addition to other polarization direction leaded to thermally astigmatic induced lens. This thermal lens affected deficiently on the laser performance by limiting the stability range of the resonator and degradation of the beam quality. Therefore, we have chosen the pump beam focused at $3 \mathrm{~mm}$ from the rod end as mentioned above to avoid bulging from the end face of the rod. Based on this principle, a long-length laser resonator was chosen in the experiment to force the laser to operate at $1053 \mathrm{~nm}(\sigma$-polarization) and terminate $1074 \mathrm{~nm}$ ( $\pi$-polarisation). Also, the resonator should be flat-flat mirrors for quenching the unfavourable transition at $1047 \mathrm{~nm}$. The simplest quenching method using one of the flat mirrors was located close to the pumped side of the laser rod and formed the short arm $L_{1}$ of the resonator. The thermal lens was approximated by a thin lens close to the pumped 
side of the Nd: YLF. The distance to the other output coupling laser mirror $M_{2}\left(L_{2}\right)$ was varied but must be always longer than $L_{1}$, bearing in mind that the optical crystal length $L_{\mathrm{YLF}} / n_{\pi}$ had to be added to the arm length that did not contain the pumped side. These resonators were distinctive in that they were only stable when the focal length of the thermal lens $f>L_{2}$. Figure 4 shows the dependence of the negative dioptric power of thermally induced lens ( $\sigma$-polarization) in Nd: YLF laser rod with pump power. The obtained value in Fig. 4 allowed us to build a flat-flat resonator and predict its stability over the wide range of pump powers before the fracture point.

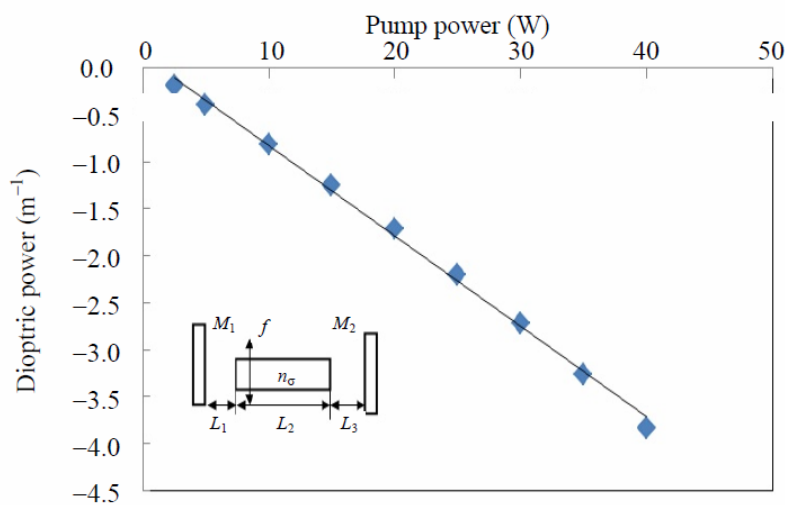

Fig. 4 Calculations of dioptric power of thermally induced lens ( $\sigma$-polarization) in Nd: YLF laser rod versus pump power.

To examine the effect of negative dioptric power of thermally induced lens on the resonator stability, we analyzed the laser operation performance by using the ABCD matrix formalism based on the standard Gaussian propagation theory after the consideration of thermal lens effect [20]. The equivalent round trip matrix is given as follows:

$$
\begin{gathered}
\mathbf{M}_{\text {roundtrip }}=\left[\begin{array}{cc}
A & B \\
C & D
\end{array}\right]= \\
{\left[\begin{array}{ll}
1 & 0 \\
0 & 1
\end{array}\right]\left[\begin{array}{cc}
1 & L_{1} \\
0 & 1
\end{array}\right]\left[\begin{array}{cc}
1 & 0 \\
\frac{-1}{f} & 1
\end{array}\right]\left[\begin{array}{cc}
1 & \frac{L_{2}}{n_{\sigma}} \\
0 & 1
\end{array}\right]} \\
{\left[\begin{array}{cc}
1 & L_{3} \\
0 & 1
\end{array}\right]\left[\begin{array}{cc}
1 & 0 \\
0 & 1
\end{array}\right]\left[\begin{array}{cc}
1 & L_{3} \\
0 & 1
\end{array}\right]} \\
{\left[\begin{array}{cc}
1 & \frac{L_{2}}{n_{\sigma}} \\
0 & 1
\end{array}\right]\left[\begin{array}{cc}
1 & 0 \\
\frac{-1}{f} & 1
\end{array}\right]\left[\begin{array}{cc}
1 & L_{1} \\
0 & 1
\end{array}\right] .}
\end{gathered}
$$

We have used for calculations, two flat mirrors $M_{1}, M_{2}, L_{1}=10 \mathrm{~mm}, L_{2}=10 \mathrm{~mm}, L_{3}=130 \mathrm{~mm}$ and variable focal length of thermally induced lens $(f)$, as sketched in the left bottom of Fig. 4.

Figure 5 shows the stability of the resonator versus the Nd: YLF thermal focal length is expressed by $(A+D) / 2$, where $-1<(A+D) / 2<1$. We could see that the laser resonator was stable before the predicted fracture limit.

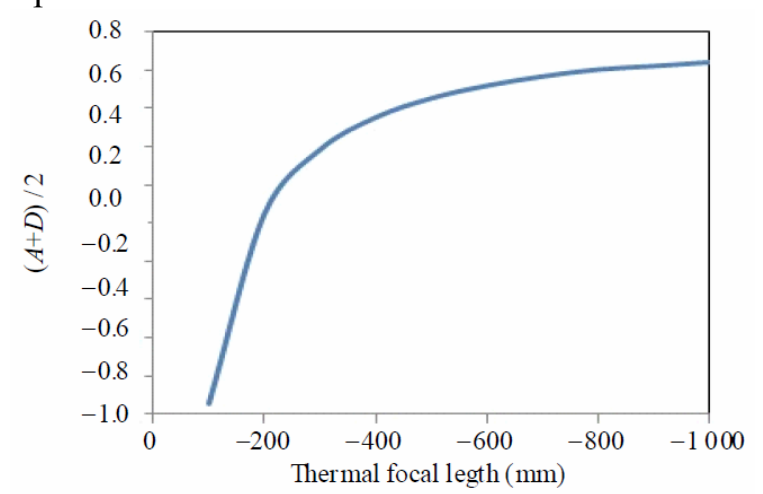

Fig. 5 Resonator stability verses thermal focal length in Nd: YLF rod.

\section{Experimental validation of EF simulations and discussion}

\subsection{Experimental setup}

The experimental setup is shown in Fig. 6. A fiber coupled laser diode (LD) was employed as a pump source. The focusing optics was configured to produce a circular beam spot of radius $\sim 300 \mu \mathrm{m}$ on the Nd: YLF laser rod. The Nd: YLF rod $10 \mathrm{~mm}$ long $(0.7 \%$ atom dopant) with $4 \mathrm{~mm}$ diameter was oriented with its $c$-axis parallel to the resonator plane. The diode wavelength was temperature detuned from the absorption peak, at $797 \mathrm{~nm}$, to longer wavelengths $805 \mathrm{~nm}$ of lower absorption to reduce the risk of rod fracture. The rod was wrapped with indium foil and mounted in a copper holder. The mounted Nd: YLF rod was water cooled at $293 \mathrm{~K}$, and the two end faces were in contact with air. The resonator geometry used was plane-plane. The input coupling mirror was with a high reflective (HR) at the wavelength near $1053 \mathrm{~nm}(R>99.5 \%)$ and high antireflective at the wavelength about $805 \mathrm{~nm}$ $(R<0.5 \%)$. The plane output coupling mirror had 
$5 \%$ transmittance at $1053 \mathrm{~nm}$. The physical length of the cavity was $150 \mathrm{~mm}$, which allowed unstable oscillation for $1047 \mathrm{~nm}$. The thermal focal length was measured by means of a Mach-Zehnder interferometer and a Shack-Hartmann sensor with a He-Ne laser beam [21-23]. The He-Ne laser beam was enlarged by a telescope to cover $4 \mathrm{~mm}$ of rod diameter and split by a beam splitter. The two arms of the Mach-Zehnder interferometer were superimposed at $45^{\circ}$ reflecting mirror of laser at $1053 \mathrm{~nm}$. The produced interference fringes which were recorded by a charge coupled device (CCD) camera. For wavefront measurements, the CCD camera was replaced by Shack-Hartmann wavefront sensor.

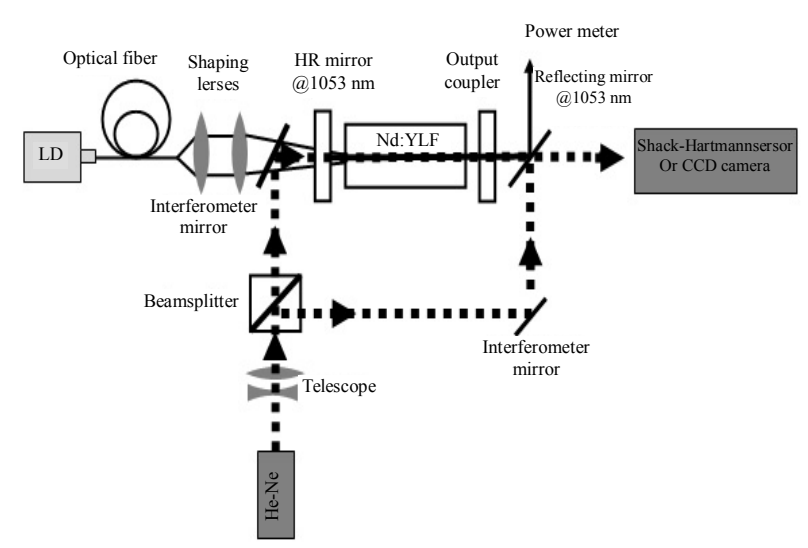

Fig. 6 Experimental setup, the dashed lines represents the $\mathrm{He}-\mathrm{Ne}$ laser beams and solid lines for laser at $1053 \mathrm{~nm}$.

\subsection{Experimental results}

At pump power of $5 \mathrm{~W}$, the output coupler was kept away from the laser crystal by adjusting the position of the output mirror. When the cavity length was $150 \mathrm{~mm}$, we obtained the laser oscillating at $1053 \mathrm{~nm}$ and suppression of laser at $1047 \mathrm{mn}$. The wavelength of the Nd: YLF laser at $1053 \mathrm{~nm}$ was measured with a half meter monochrometer 1200 groves/mm and detected by silicon photodiode connected with a phase-lock amplifier and chopper wheel not shown in Fig. 6.

Figure 7 shows the laser output power of Nd: YLF versus pump power. The threshold was achieved $2 \mathrm{~W}$ of incident pump power. The emitted laser spectrum centered at $1052.8 \mathrm{~nm}$ with full width at half maximum (FWHM) of $\sim 3.5 \mathrm{~nm}$. The maximum obtained output power was $6.8 \mathrm{~W}$ under a pump power of $30 \mathrm{~W}$ from the laser diode. The optical-to-optical conversion efficiency at the maximum power level was approximately $24.8 \%$.

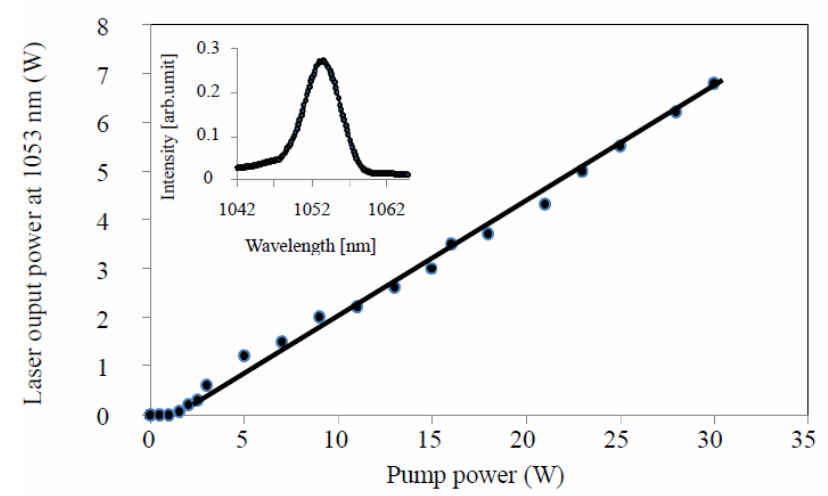

Fig. 7 Laser output at $1052.8 \mathrm{~nm}$ versus pump power, the top left if the laser spectrum measurement.

Figure 8 shows the deformation of the phase front that occurred when the $\mathrm{He}-\mathrm{Ne}$ laser beam propagated the Nd: YLF laser rod pumped at $15 \mathrm{~W}$ of pump powers. Since most of the pump power is concentrated in the center of the rod, the lens near the center of the rod is stronger as compared with the lens in the outer region. The dashed lines of Fig. 8(b) represent the fitting of the parabola presented in the following equation:

$$
z(x, y)=a \cdot x+b \cdot y+c \cdot x \cdot y+d \cdot x+e \cdot y+f .
$$

The dioptric power of the thermal lens is proportional to the curvature of parabola and is proportional to coefficients $a$ and $b$. Depending on the pump power distribution in the laser rod, the thermal lens may not be radically symmetric, which leads to an asymmetric thermal lens with two thermal powers $D_{x}$ and $D_{y}$.

Comparison between the calculated negative thermal lens power and the experimental measurements for Nd: YLF laser rod is presented in Fig. 9. The deviation between measured and calculated data refers to the accuracy of the FE analysis which is given by two points, namely the numerical resolution of the algorithm and the values of the input parameters. 


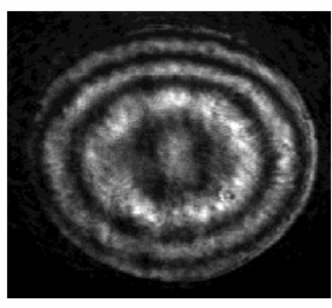

(a)

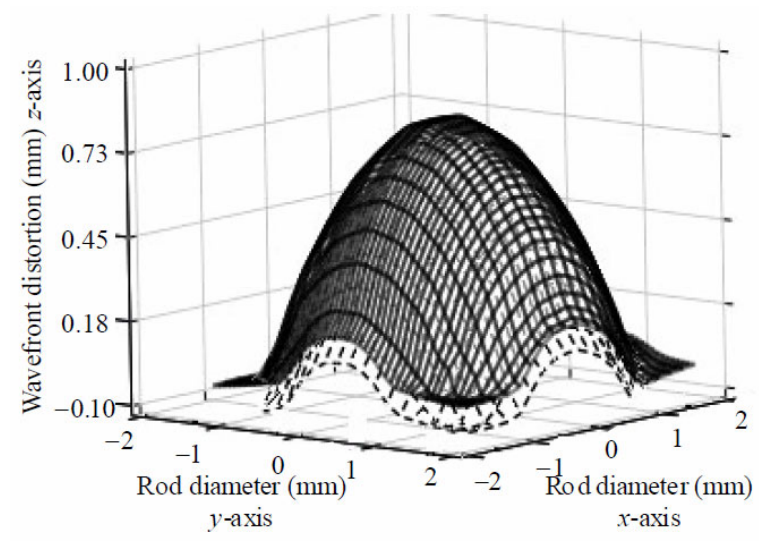

(b)

Fig. 8 Deformation in wavefront of He-Ne laser beam after passing through pumped Nd: YLF laser rod at $15 \mathrm{~W}$ measured with (a) Mach-Zehnder interferometer and (b) Shack-Hartmann sensor (solid lines), the fitting is represented by he dashed lines.

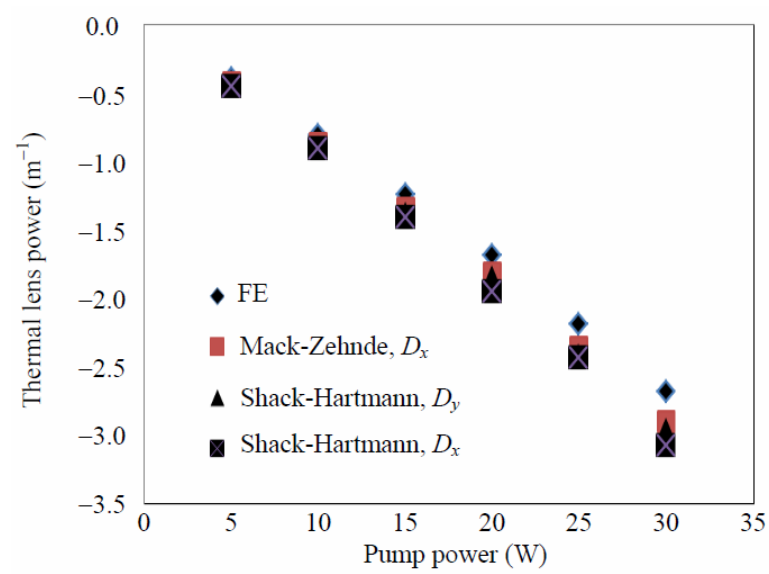

Fig. 9 Comparison between FE simulations and experimental measurements.

\section{Conclusions}

In conclusion, we have presented an analysis of thermal effect in diode pumped Nd: YLF laser rod and its lasing characteristics at $1053 \mathrm{~nm}$ under continuous-wave operation. The temperature and stress intensity distributions in the Nd: YLF laser rod were shown by using LASCAD software. The central temperature and stress intensity of Nd: YLF laser rod verses pump powers was presented with the clear linear dependence. The thermal focal length was calculated for the laser emission at $1053 \mathrm{~nm} \sigma$-polarisation and utilized to design the laser resonator. The direct measurement of thermal lens powers using the Mach-Zehnder interferometer and Shack-Hartmann wavefront sensor at different pump powers was introduced. The experimental measurements were coinciding with the numerical simulations. The maximum output power of $6.8 \mathrm{~W}$ was obtained.

\section{Acknowledgment}

The authors grateful thank the laboratories facilities of quantum optics research group (QORG) at Taif University KSA.

Open Access This article is distributed under the terms of the Creative Commons Attribution 4.0 International License (http://creativecommons.org/ licenses/by/4.0/), which permits unrestricted use, distribution, and reproduction in any medium, provided you give appropriate credit to the original author(s) and the source, provide a link to the Creative Commons license, and indicate if changes were made.

\section{References}

[1] W. Koechner and M. K. Wilson, "Solid-state laser engineering: second completely revised and updated edition," Applied Optics, 1989, 28(48): 509-530.

[2] A. K. Cousins, "Temperature and thermal stress scaling in finite-length end-pumped laser rods," IEEE Journal of Quantum Electronics, 1992, 28(4): 1057-1069.

[3] S. C. Tidwell, J. F. Seamans, and M. S. Bowers, "Highly efficient 60-W TEM00 cw diode-endpumped Nd: YAG laser," Optics Letters, 1993, 18(2): 116-118.

[4] J. E. Murray, "Pulsed gain and thermal lensing of Nd: LiYF4," IEEE Journal of Quantum Electronics, 2003, 19(4): 488-491.

[5] C. Keileck, A. Hirth, and M. T. Schellhorn, "Performances of Ho: YAG laser intracavity-pumped by a diode-pumped TM: YLF laser," SPIE, 2004, 5478: 488-491.

[6] C. Keileck, A. Hirth, and M. T. Schellhorn, "Performances of Ho: YAG laser intracavity-pumped by a diode-pumped TM: YLF laser," Optics Letters, 2003, 28: 1933-1935.

[7] W. Koechner and D. K. Rice, "Effect of birefringence 
on the performance of linearly polarized YAG: Nd lasers," IEEE Journal of Quantum Electronics, 1970, 6(9): 557-566.

[8] Y. J. Huang, C. Y. Tang, W. L. Lee, Y. P. Huang, S. C. Huang, and Y. F. Chen, "Efficient passively Q-switched Nd: YLF TEM00-mode laser at $1053 \mathrm{~nm}$ : selection of polarization with birefringence," Applied Physics B, 2012, 108(2): 313-317.

[9] K. Schuhmann, K. Kirch, F. Nez, R. Pohl, and A. Antognini, "Thin-disk laser scaling limit due to thermal lens induced misalignment instability," Applied Optics, 2016, 55 (22): 9022-9032.

[10] D. L. Kim, B. T. Kim, "Laser output power losses in ceramic Nd: YAG lasers due to thermal effects," Optik-International Journal for Light and Electron Optics, 2016, 127(20): 9738-9742.

[11] S. Chenais, F. Druon, F. Balembois, G. Lucas-Leclin, Y. Fichot, P. Georges, et al., "Thermal lensing measurements in diode-pumped $\mathrm{Yb}$ doped GdCOB, YCOB, YSO, YAG and KGW," Optical Materials, 2003, 22(2): 129-137.

[12] S. Chenais, F. Balembois, F. Druon, G. Lucas-Leclin, and P. Georges, "Thermal lensing in diode-pumped ytterbium lasers part II: evaluation of quantum efficiencies and thermo-optic coefficients," IEEE Journal of Quantum Electronics, 2004, 40(9): 1235-1242.

[13] P. J. Hardman, W. A. Clarkson, G. J. Friel, M. Pollnau, and D. C. Hanna, "Energy-transfer upconversion and thermal lensing in high-power end-pumped Nd: YLF laser crystals," IEEE Journal of Quantum Electronics, 1999, 35(4): 647-655.

[14] Z. L. Zhang, Q. Liu, M. M. Nie, E. C. Ji, and M. L. Gong, "Experimental and theoretical study of the weak and asymmetrical thermal lens effect of
Nd:YLF crystal for $\sigma$ and $\pi$ polarizations," Applied Physics B, 2015, 120(4): 689-696.

[15] LASCAD Gmbh, Brimhildenstr. 9, 80639 Munich, Germany. Available online: http://www.las-cad. com/index.php.

[16] D. C. Brown, "Ultrahigh-average-power diodepumped Nd: YAG and Yb: YAG lasers," IEEE Journal of Quantum Electronics, 1977, 33(5): 861-873.

[17] C. Bollig, C. Jacobs, M. J. D. Esser, E. H. Bernhardi, and H. M. von Bergmann, "Power and energy scaling of a diode-end-pumped Nd: YLF laser through gain optimization," Optics Express, 2010, 18(13): 13993-14003.

[18] M. J. Weber, Hand book of pptical materials. New York: CRC Press London, 2002: 1-499.

[19] N. U. Wetter, E. C. Sousa, F. A. Camargo, I. M. Ranieri, and S. L. Baldochi, "Efficient and compact diode side pumped Nd: YLF laser operating at 1053 nm with high beam quality," Journal of Optics A Pure \& Applied Optics, 2008, 10(10): 1-5.

[20] M. E. Innocenzi, H. T. Yura, C. L. Fincher, and R. A. Fields, "Thermal modeling of continuous-wave end-pumped solid-state lasers," Applied Physics Letters, 1990, 56(19): 1831-1833.

[21] Y. Liu, W. Zhang, T. Xu, J. He, F. Zhang, and F. Li, "Fiber laser sensing system and its applications," Photonic Sensors, 2011, 1(1): 43-53.

[22] M. Mayeh and F. Farahi, "Laser beam shaping and mode conversion in optical fibers," Photonic Sensors, 2011, 1(1): 187-198.

[23] P. G. Jia, G. C. Fang, and D. H. Wang "Characterization of miniature fiber-optic Fabry-Perot interferometric sensors based on hollow silica tube," Photonic Sensors, 2016, 6(3): 193-198. 